\title{
Infliximab in the treatment of severe ulcerative colitis
}

The treatment of ulcerative colitis shows a therapeutic window that is of great relevance for daily practice: the management of steroid-dependent patients who do not respond to mercaptopurine and that of steroid-refractory individuals. In a paper published in this issue, F. Bermejo et al. (1) manage these patients with infliximab (anti-TNF- $\alpha$ ) in an attempt to solve this fortunately not-so-frequent difficulty.

TNF- $\alpha$ is synthesized by macrophages and to a lesser extent by T cells, NK cells, and mast cells within the colonic mucosa. The primary functions of this proinflammatory cytokine include: induction of class II major histocompatibility complex in association with IFN- $\gamma$ in colonocytes, inhibition of enterocyte proliferation in the mouse, metalloproteinase synthesis stimulation, IL-8, VAN-1 and ICAM-1 synthesis activation synergically with IFN- $\gamma$, increased permeability of the mucosal barrier, MCP-3-mediated stimulation of chemotaxis in monocytes, neutrophils, eosinophils and basophils, and inhibition of $\mathrm{T}$ cell apoptosis (2-6).

In August 1998, the FDA approved infliximab for the treatment of fistulous inflammatory Crohn's disease. This was the first chimeric antibody to neutralize TNF- $\alpha$. The results of phase 3 treatment were so much brilliant in "healing" both gross and microscopic lesions that not more than 200 patients were ultimately needed, to which the drug's orphan status contributed.

Crohn's disease and ulcerative colitis are two distinct conditions that share a number of aspects, one of them being TNF- $\alpha$ as a key element in their inflammatory cascades, even though their intimate mechanisms differ. Evidence exists for the latter statement. On the one hand this cytokine is known to be highly concentrated in the lesions, circulation and feces of patients with colitis, and its levels correlate with inflammatory activity outbreaks (7-9). On the other hand anti-TNF- $\alpha$ therapy obtained positive results in ulcerative colitis animal models (10).

Research on the therapeutic role of infliximab in ulcerative colitis is and has been very poor. During the last 5-6 years a number of observational studies emerged on the treatment of ulcerative colitis with this anti-cytokine, and all of them supported a percentage of therapeutic response of $60-80 \%$, similar to that seen for Crohn's disease; however, they are based on hardly comparable, much too short, non-randomized series with no control groups and heterogeneous patient groups -including patients with pouchitis, indeterminate colitis, and both steroid-dependent and steroid-refractory severe colitis- as well as follow-up periods of 4 to 12 months in duration, much too wide relapse intervals, and scarce information on patients who keep their colon at follow-up endpoint (6,11-15). In 2003 the first extensive, randomized, controlled study in a group of steroid-refractory patients is reported, and no therapeutic effects are found with two $5 \mathrm{mg} / \mathrm{kg}$ doses at $0-2$ weeks after a second infusion of infliximab (16). 
The histologies and inflammatory models of ulcerative colitis and Crohn's disease differ. Is the role of TNF- $\alpha$ within the inflammatory cascade also different in these two conditions? Do patients with ulcerative colitis make up a homogeneous group? Is there a reason to believe that steroid-refractory patients respond in a poorer way when compared to steroid-dependent patients? $(15,16)$. In the article by $\mathrm{F}$. Bermejo et al. (1), one patient did not respond but his/her refractory status is not specified; we would rather think this patient was refractory to steroids. Response to anti-TNF- $\alpha$ agents may not be accurately assessed until extensive randomized, controlled therapeutic trials are designed that include homogeneous patient groups, but the potential therapeutic niche of such drugs may be certainly delimited (17).

By using mesalazine, steroids and 6-mercaptopurine we may control ulcerative colitis in more than $90 \%$ of patients, but patients are either steroid-dependent or steroid-refractory in a percentage that is difficult to establish, and mercaptopurine is unable to solve this due to either prolonged therapeutic latency or intolerance; we then face a surgical indication that is pressing on occasion and leads to patient mutilation, with no assurance that the "disease" will be cured, and in association with pouchitis and significant morbidity and mortality.

Although suggested options include agents such as tacrolimus (FK506), intravenous 6-mercaptopurine, or lymphokine antagonists, well-researched data exist for cyclosporine (CyA) alone, a drug that inhibits IL-2 receptors on T cells and IL-2 secretion, as well as the secretion of IL-3, IL-4, and IFN- $\gamma$ in a secondary manner.

Using 4-5 mg/kg/day intravenously, patients with UC exhibit blood levels ranging from $120 \mathrm{ng} / \mathrm{mL}$ to $483 \mathrm{ng} / \mathrm{mL}$; by keeping concentrations in the high range end, patients manage to keep their colon at six months in a percentage oscillating between 33 and $80 \%$, depending on whether medication is combined with mercaptopurine (18-21). These figures closely resemble those reported for infiximab.

There is seemingly no doubt that infliximab manages to nip severe ulcerative colitis flares in the proverbial bud, but its use is restricted to a marginal population of patients: those steroid-dependent individuals who do not respond to mercaptopurine. To steroid-refractory patients, too? How many infusions are needed to elicit response or otherwise consider a patient treatment-refractory? Are there any predictive factors for response? The one key question is whether this is more effective and less toxic when compared to CyA or tacrolimus followed by mercaptopurine, since it is certainly not cheaper.

J. Maté Jiménez

Gastroenterology Unit. Hospital de La Princesa. Madrid, Spain

\section{REFERENCES}

1. Bermejo F, López-Sanromán A, Hinijosa J, et al. Infliximab induce respuesta clínica, endoscopica e histológica en la Colitis Ulcerosa. Rev Esp Enferm Dig 2004; 96 (2): 94-101.

2. HorieY, Chiba M, Suzuki T, et al. Inducción of major histocompatibility complex class II on human colonic ephitelium by Interferon-gamma, Tumor necrosis factor-alpha and Interleukin-2. J Gastroenterol 1998; 33: $30-47$

3. Kaiser GC, Polk DB. Tumor necrosis factor-alpha proliferation in a mouse intestinal cell line. Gastroenterology. 1997; 112: 1231-40. 
4. Pender SLF, Fell JME, Chamow SM, et al. A major role for matrix metalloproteinases in T cell injury in the gut (abstract). J Immunol 1997; 1580: 82.

5. Fish SM, Proujuansky R, Reenstra WW. Synergy effects of IFN-g and Tumor necrosis factor-alpha on T8/4 function. Gut 1999; 45: 191-8.

6. Lichtenstein GR. Is infliximab effective for induccion of remission in patients with ulcerative colitis? Inflammatory Bowell Diseases 2001; 71: 89-93.

7. Ishiguru Y. Mucosal proinflammatory cytokines production correlates with endoscopic activity of ulcerative colitis. J Gastoenterol 1999; 34: 66-74.

8. Guimbaud R, Bertand Y, Chauvelot-Moachon L, et al. Network of inflammatory citokines and correlation with disease activity in ulcerative colitis. Am J Gastroenterol 1998; 93: 2397-404.

9. Suiki T, Mitsuyama K, Toyonaga A, et al. Detection of pro and anti-inflammatory cytokines in stools of patiens with inflammatory bowel disease. Scand J Gastroenterol 1998; 33: 616-22.

10. Watkins PE, Warren BF, Stiphens S et al. Treatment of ulcerative colitis in the cotton-top tamarin using antibody to tumor necrosis factor alpha. Gut 1997; 40: 628-33.

11. Sands BE, Tremaine W, Sandborn WJ, et al. Infliximab in the treatment of severe, steroid-refractary ulcerative colitis: A pilot study. Inflamm Bowell Dis 2001; 7: 83-8.

12. Chey WY. Infliximab for patients with refractory ulcerative colitis 2001; 7 (Supl. 1): 30-33.

13. Gornet JM, Couve S, Hassani Z, et al. Infliximab for refractory ulcerative colitis or indeterminate colitis: an open-label multicentre study. Aliment Pharmacol Ther 2003; 15: 175-81.

14. Viscido A, Habib FI, Kohn A, et al. Infliximab in refractory pouchitis complicated by fistulae following ileoanal pouch for ulcerative colitis. Aliment Pharmacol Ther 2003; 18: 1263-71.

15. Su C, Salzberg BA, Lewis JD, et al. Efficacy of anti-tumor necrosis factor therapy in patients with ulcerative colitis. Am J Gastroenterol 2002; 97: 2577-84.

16. Probert CS, Hearing SD, Schreiber S, et al. Infliximab in moderately severe glucocorticoid resistant ulcerative colitis: a randomised controlled trial. Gut 2003; 52: 998-1002.

17. Rutgeerts P. Imfliximab for uncerative colitis: The need for adequately powered placebo-controlled trials. Am J Gastroenterol 2002; 97: 2488-9.

18. Lichtiger S, Present DH, Kornbluth A, et al. Cyclosporine in severe ulcerative colitis refractory to steroid therapy. N Engl J Med 1994; 330: 1841-5.

19. Carbonell F, Boruchowicz A, Duclos B, et al. Intravenous cyclosporine in attacks of ulcerative colitis. Dig Dis Sci 1996; 41: 2471-6.

20. Fernández-Bañares F, Bertrán X, Esteve-Comas M, et al. Azathioprine is useful in maintaining long-term remission induced by cyclosporine in steroid-refractory severe ulcerative colitis. Am J Gastroenterol 1996; 91 : 2498-9.

21. Hermida-Rodríguez C, Cantero-Perona J, García-Valribera R, Pajares-García JM, Maté-Jiménez J. High doses cyclosporine for steroid-refractary attacks of Intestinal Bowel Disease. Hepato-Gastroenterology 1999; 46: $2265-8$.

\section{Infliximab en el tratamiento de la colitis ulcerosa grave}

En el tratamiento de la colitis ulcerosa existe una ventana terapéutica de gran importancia en la clínica diaria: el tratamiento de los enfermos córtico-dependientes que no responden a la mercaptopurina y los córtico-refractarios. En un trabajo que se publica en este número Bermejo y cols. (1) tratan a estos enfermos con infliximab (anti-TNF- $\alpha$ ) en un intento de solventar este problema que afortunadamente no es demasiado frecuente.

El TNF- $\alpha$ se sintetiza en los macrófagos y en menor medida en linfocitos T, células NK y en los mastocitos de la mucosa del colon. Esta citoquina proinflamatoria tiene como funciones principales: la inducción del complejo mayor de histocompatibilidad -en asociación con el IFN- $\gamma$ - de clase II en los colonocitos, la inhibición de la proliferación de los enterocitos del ratón, la estimulación en la síntesis de metal- 
proteinasas, el estímulo de la síntesis de IL-8, VAN-1 e ICAM-1, en sinergia con el IFN- $\gamma$, el aumento de la permeabilidad de la barrera mucosa, la estimulación de la quimiotaxis de monocitos, neutrófilos, eosinófilos y basófilos mediada por la MCP3, y la inhibición de la apoptosis de los linfocitos T (2-6).

En agosto del 98 la FDA aprueba el infliximab para el tratamiento de la enfermedad de Crohn fistulosa e inflamatoria. Es el primer anticuerpo quimérico que neutraliza el TNF- $\alpha$. Los resultados del tratamiento en fase 3 fueron tan brillantes, en la "curación" macro y microscópica de las lesiones, que no fueron necesarios más de 200 enfermos, ayudando su condición de fármaco huérfano.

La enfermedad de Crohn y la colitis ulcerosa son dos entidades distintas aunque emparentadas en algunos puntos, y uno de ellos es que su cascada infamatoria -aunque difieran en su íntimo mecanismo- tienen en el TNF- $\alpha$ su clave fundamental. Existen evidencias que permiten la afirmación anterior. Por un lado se conocen las elevadas concentraciones de esta citoquina en las lesiones, la circulación y las heces de los enfermos de colitis, y que estos niveles se correlacionan con los brotes de actividad inflamatoria (7-9). Por otro lado se conocen los buenos resultados del anti-TNF- $\alpha$ en los modelos animales de colitis ulcerosa (10).

La investigación sobre el papel terapéutico del infliximab en la colitis ulcerosa ha sido, y es, muy pobre. Desde hace 5-6 años aparecen algunos estudios observacionales sobre el tratamiento de la colitis ulcerosa con esta anti-citoquina, y todos apoyan la idea de que el porcentaje de respuesta terapéutica es del 60-80\%, similar al observado en la enfermedad de Crohn, pero se basan en series muy cortas sin randomizar, sin grupos controles, difícilmente comparables, con grupos de enfermos muy heterogéneos -se mezclan puchitis, colitis indeterminadas, colitis graves con córtico-dependientes y córtico-refractarias- periodos de seguimiento que oscilan entre los 4 y los 12 meses, una horquilla de recidivas muy amplia y pobre información sobre los enfermos que conservan su colon al final del seguimiento (6,11-15). En el 2003 aparece el primer estudio extenso, en un grupo de enfermos córtico-refractarios, controlado y randomizado que no encuentra ningún efecto terapéutico con dos dosis de $5 \mathrm{mg} / \mathrm{kg}$ a las 0-2 semanas de la segunda infusión de infliximab (16).

La histología y el modelo inflamatorio de la colitis ulcerosa y de la enfermedad de Crohn son diferentes. ¿Lo es también el papel del TNF- $\alpha$ en la cascada inflamatoria de ambas entidades? ¿Son los enfermos de colitis ulcerosa un grupo homogéneo? Existen indicios que hacen pensar que los enfermos córtico-refractarios responden peor que los córtico-dependientes $(15,16)$. En el trabajo de Bermejo y cols. (1) uno de los enfermos no responde al tratamiento, no se especifica si era el córtico-refractario, nos gustaria pensar que sí. No sabremos con certeza la respuesta a los anti-TNF- $\alpha$ hasta que se diseñen extensos ensayos terapéuticos randomizados, controlados y con grupos de pacientes homogéneos, pero si podemos acotar su posible nicho terapéutico (17).

Con la mesalacina, los estorides y la 6-mercaptopurina podemos controlar la colitis ulcerosa en más de $90 \%$ de los enfermos, pero en un porcentaje difícil de concretar los enfermos se muestran córtico-refactarios o córtico-dependientes sin que la mercaptopurina pueda resolver la situación -bien sea por su larga latencia terapéutica o por intolerancia- y estamos ante una indicación quirúrgica, a veces perentoria, que termina con la mutilación del enfermo sin garantías de acabar con la "enfermedad", que llevará aparejada una "puchitis", una elevada morbilidad y mortalidad no despreciable. 
Aunque son varias las alternativas propuestas: tracolimus (FK506), 6-mercaptopurina intravenosa, antagonistas de las linfoquinas, tan sólo tenemos contrastada información sobre la ciclosporina (CyA) que es una droga que actúa inhibiendo los receptores de la IL-2 de los linfocitos T, la secreción de la misma y de forma secundaria inhibiendo la secreción de IL-3, IL-4, e IFN- $\gamma$.

Con 4-5 mg/kg/día por vía intravenosa los enfermos de CU alcanzan niveles entre 120 y $483 \mathrm{ng} / \mathrm{ml}$ en sangre y manteniendo las concentraciones en la parte alta de la horquilla los enfermos consiguen conservar su colon a los seis meses en unos porcentajes que varían entre el 33 y el 80\%, según se combine esta medicación con mercaptopurina o no (18-21). Cifras muy similares a las publicadas para el infiximab.

Parece indudable que el infliximab consigue yugular el brote grave de colitis ulcerosa, pero su uso estará restringido a una población marginal de enfermos; los córtico-dependientes que no respondan a la mercaptopurina. ¿También a los córtico-refractarios? ¿Cuántas infusiones son necesarias para obtener un respuesta o considerar al enfermo refractario al tratamiento? ¿Existen factores predictivos de respuesta? La pregunta clave es si será más eficaz y menos tóxica que la CyA o el tracolimus seguidos de mercaptopurina, porque más barato no parece. 